\title{
1 Comparison of surface extraction techniques performance in computed tomography for 3D 3 complex micro-geometry dimensional measurements
}

\author{
Marta Torralba ${ }^{1}$, Roberto Jiménez ${ }^{1}$, José A. Yagüe-Fabra ${ }^{2, *}$, Sinué Ontiveros ${ }^{3}$, Guido Tosello ${ }^{4}$ \\ 1 Centro Universitario de la Defensa, A.G.M. Carretera Huesca s/n, 50090 Zaragoza; martatg@unizar.es; \\ rjimenez@unizar.es \\ 2 I3A, Universidad de Zaragoza, María de Luna 3, 50018 Zaragoza; jyague@unizar.es; ORCID: \\ 0000-0001-7152-4117 \\ 3 Department of Industrial Engineering, Autonomous University of Baja California, Mexico; \\ sinue.ontiveros@uabc.edu.mx \\ 4 Department of Mechanical Engineering, Technical University of Denmark, Kgs. Lyngby, Denmark; \\ guto@mek.dtu.dk \\ * Correspondence: jyague@unizar.es; Tel.: +34-976-762-561
}

\begin{abstract}
The number of industrial applications of Computed Tomography (CT) for dimensional metrology in $10^{0}-10^{3} \mathrm{~mm}$ range has been continuously increasing, especially in the last years. Due to its specific characteristics Computed Tomography has the potential to be employed as a viable solution for measuring 3D complex micro-geometries as well (i.e. in the sub-mm dimensional range). However, there are different factors that may influence the CT process performance, being one of them the surface extraction technique used. In this paper two different extraction techniques are applied to measure a complex miniaturized dental file by $\mathrm{CT}$ in order to analyze its contribution to the final measurement uncertainty in complex geometries at the $\mathrm{mm}$ to sub-mm scales. The first method is based on a similarity analysis: the threshold determination; while the second one is based on a gradient or discontinuity analysis: the 3D Canny algorithm. This algorithm has proven to provide accurate results in parts with simple geometries, but its suitability for 3D complex geometries has not been proven so far. To verify the measurement results and compare both techniques, reference measurements are performed on an optical coordinate measuring machine (OCMM). The systematic errors and uncertainty results obtained show that the 3D Canny adapted method slightly lower systematic deviations and a more robust edge definition than the local threshold method for 3D complex micro-geometry dimensional measurements.
\end{abstract}

Keywords: 3D complex geometry; Computed Tomography; Surface extraction; Canny algorithm

\section{Introduction}

The geometrical complexity of industrial components with micro three-dimensional features has been rapidly increasing in the last years. That implies a parallel effort from the metrology point of view in order to assure the correct dimensional measurement and tolerance verification of these parts [1]. Tactile and optical techniques are available to perform length measurements in three dimensions with high accuracy. However, they exhibit limitations when measuring 3D complex geometries, especially at sub-mm scale [2-4]. Tactile techniques are limited in terms of accessibility and minimum measurable feature size due to the probe and stylus dimensions, measuring point density and deformation of high aspect ratio structures under measurement and of soft substrate materials due to the probing force. Non-contact techniques, such as interferometric microscopes [5] or laser line scanning [6] have limitations both in measuring vertical walls and high aspect ratio structures, due to surface properties and accessing out-of-sight features. In recent years, 3D imaging by means of Computed Tomography (CT) has emerged as a new technology for industrial quality control in many industrial applications [7]. The main metrological capability of this non-contact 
imaging technique is based on the possibility of acquiring a densely populated 3D scanning point cloud of an object, allowing the measuring of free-form surfaces [8], non-accessible internal structures $[9,10]$ and even multi-material components [11-13]. Therefore, regarding 3D complex surface geometries, Computed Tomography has the potential to become a viable solution for their dimensional measuring. However, CT metrology improvements have been initially focused on the measurement of reference standards and industrial parts that are characterized by simple or regular geometries, i.e. intrinsically linear or approximated by linear forms (lines, planes, circles, spheres, cylinders, etc.) [14-16] and the study and optimization of this technique for 3D complex micro-geometry dimensional measurements has not been addressed so far.

The main disadvantage of $\mathrm{CT}$ is the high number and the complexity of the factors related to hardware, software, environment, workpiece and operator that may influence the system performance [17-19]. Previous works [17-19] have already addressed the difficulty of identifying and quantifying all the uncertainty sources that should be considered for a measurement uncertainty evaluation. In addition, research has been carried out to demonstrate and evaluate the contribution of specific factors with regards to metrology issues: for instance, the work presented in [20] is focused on those influencing factors that can be controlled by the machine operator (e.g. magnification of the workpiece, number of projections, position and orientation of the workpiece). Simulated computed tomography data is used in [21] to investigate the effect of angular misalignments of a flat-panel detector, and in [22] for studying the influence factors on image quality and scanning geometry by numerical generation modelling of $\mathrm{X}$-ray projections. A more extensive review of geometrical influence factors is outlined by Ferrucci et al. in [23] with respect to the geometrical offsets and misalignments of the cone-beam CT system. Hiller et al. compared the results when measuring a test object with two CT systems, two STL models provided by each of the scanners and two different software packages for geometrical fitting [24]. Different measuring strategies are also compared in [25], where three different inspection software packages for volume and surface data analysis were applied. Additionally, the authors in [26] evaluated and quantified the repeatability of post-processing settings, such as data fitting, the definition of the datum system and surface determination, which is also analyzed in $[27,28]$. As some of these works show, the surface extraction technique used is one of the most influent factors in the final measurement uncertainty.

All these studies have been also carried out using parts with simplified geometrical shapes to be analyzed. However, when the influences of these factors are to be studied on 3D free-form geometries or complex shapes, and particularly when they belong to micro-components, their evaluation becomes more complicated. The direct comparison between calibrated values and measured values of 3D complex geometries is more challenging than for, for example, spheres, cylinders, etc. The goal of the present work is to study the influence of two different surface extraction techniques in the final systematic error and measurement uncertainty when applied to measure a complex miniaturized component for medical applications (dental endodontic file) by CT. The first method is based on the threshold determination strategy [29,30], widely used in commercial CT systems and based on the similarity principle. The second one is based on a discontinuity analysis by applying the 3D Canny adapted algorithm developed by the authors in [31]. Both methods have been previously studied by the authors in order to, firstly, analyze advantages and drawbacks of using CT metrology in comparison with other measuring systems in micro-molded parts with regular geometries [29] and, secondly, carry out a mutual comparison of both surface extraction techniques applied to parts or reference standards also with regular geometries [30-32]. In all these previous works reference calibration objects with regular geometries were used. The same types of reference objects with regular geometries are found in the literature published for other authors [23-27]. In the present work the authors propose a real object with complex 3D geometry, which is an innovation with respect to the previous works found in the literature. This presents some challenges, especially for the 3D Canny algorithm since, as described in [31] it uses an strategy that analyses the surface from the three main Cartesian directions in order to extract the surface. That was proven to be effective with regular surfaces than can be easily 
defined along those Cartesian axes [31]. However, in 3D complex geometries, which are not necessarily aligned with those Cartesian axes, the effectiveness of these algorithms has to be analyzed, what becomes the main objective and novelty of the present work.

To verify the CT measurement results and compare both methods, the dental file is also characterized by an optical CMM (OCMM). Hence, the paper is organized as follows. Firstly, Section 2 introduces the workpiece and workflow applied in dimensional CT metrology, the description of the surface extraction methods and the common measurement strategy considered for the OCMM and the CT systems. In Section 3, the measurement results are presented. The systematic error analysis and the uncertainty estimation for both OCMM and CT measurements are included, also describing the assessment of the CT system tolerance verification capability in order to compare the results of both surface extraction techniques. The article ends in Section 4 with the conclusions about the strong points and weaknesses of both techniques when they are applied to the geometrical measurement of 3D complex shapes of micro-components.

\section{Materials and Methods}

\subsection{Workpiece: $3 D$ complex geometry dental file}

A complex miniaturized component for medical applications, a dental file [33,34], was considered for this study. The ProTaper F2 finishing file (produced by Dentsply Maillefer, York, PA, USA) is made of Nickel Titanium (Ni-Ti) alloy and presents complex helix geometry, due to its variable sub-mm diameter, and variable helix pitch and helix angle along its axis. Figure 1 shows a detail of the active cutting part of the file. Its measurands are defined according to ISO 3630-1:2008 [35], being the following (see Figure 2):

- $\quad$ Length of the active cutting part (La).

- Variable diameter along the file length (Dn, $\mathrm{n}=0,1,2, \ldots, 12)$.

- Helix angle $(\mathbf{H n}, \mathrm{n}=1, \ldots, 9)$ or the angle formed between the helix and the file axial axis.

- Helix pitch (Pn, n=1,..,9) or the distance between a point in the forward edge and its corresponding point in the adjacent edge along the file longitudinal axis.

The diameter Dr is used as a reference value for the surface extraction techniques since it can be easily calibrated by tactile methods. The standard [35] specifies nominal values for the cutting segment (La, $16 \mathrm{~mm}$ length); tip diameter (D0, $0.25 \mathrm{~mm}$ ); fixed conicity ( $8 \%$ between D0 and D3); variable conicity from D3 to D12 along its axis; and a maximal flute diameter (Dr, $1.20 \mathrm{~mm}$ ). Other dimensional features are specified neither by the standards, nor by the manufacturer, so that the tolerances used in this work are based only on the previously mentioned. For diameters from D0 to D6, their tolerance is $\pm 20 \mu \mathrm{m}$. For diameters from D7 to D12, the specified tolerance is $\pm 40 \mu \mathrm{m}$. For the active cutting length (La) the tolerance is $\pm 0.5 \mathrm{~mm}$.

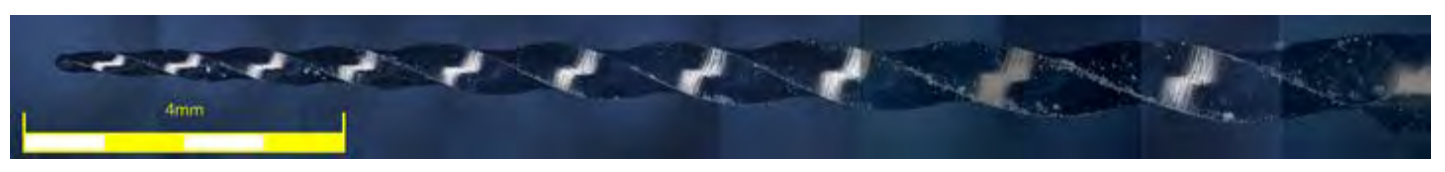

Figure 1. Dental file workpiece: detailed view of the active cutting part with complex helix geometry and variable sub-mm diameter. Image obtained from the OCMM. 

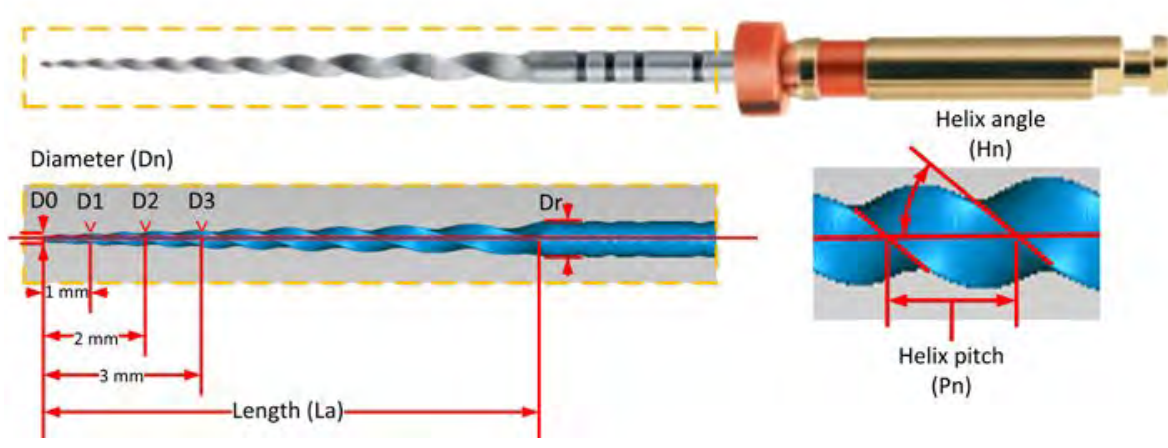

Figure 2. Dental file workpiece: characteristic dimensions to be verified by computed tomography La (length), Dn (diameter), Pn (helix pitch) and Hn (helix angle).

\subsection{Dimensional CT metrology workflow}

As mentioned before, the metrological capability of CT systems is limited by the numerous and complex factors which influence the system performance. In the literature, the measurement error sources have been classified by different criteria $[18,19]$. In brief, the main factors are the following:

- CT-system or hardware (X-ray source, rotary table, detector, global CT-scan geometry, etc.).

- Software and data processing (reconstruction algorithm, surface detection methods, data correction, etc.).

- Environment (temperature, humidity, vibrations, etc.).

- Workpiece (geometry, material, manufacturing variations, surface roughness, etc.).

- Operator (scanning parameters, experience, etc.).

These influencing factors are present in the different required steps in CT measurements. These phases and the typical process chain of dimensional measurement by means of $\mathrm{CT}$ are schematized in Figure 3. First, the 2D X-ray scans provide the projected images of the measured workpiece. Secondly, the images are reconstructed into a 3D voxel model. Then, the segmentation phase allows distinguishing the edges from the point cloud of the workpiece by using surface extraction algorithms. To conclude, dimensions of measurands are determined by a fitting procedure. It is after this final phase when the evaluation of the results can be carried out, including the measurement uncertainty estimation. In this work, the different parameters of the dental file were measured both by the CT scanner and, previously, by an optical coordinate measuring machine (OCMM) as a reference in order to be able to carry out a result comparison with a calibrated measuring system.

Recent research demonstrates that the measurement uncertainty value is mainly affected by both the post-processing strategy and the user influence [36]. Thus, the post-processing phase can be considered one of the key phases in terms of uncertainty evaluation. Therefore, two surface extraction techniques are applied in this work in order to compare them by analyzing the results obtained when measuring a miniaturized dental file having a 3D complex geometry. Both methods briefly described later are the following: CT1 or local threshold method [29] and CT2 based on the 3D Canny algorithm [31]. 


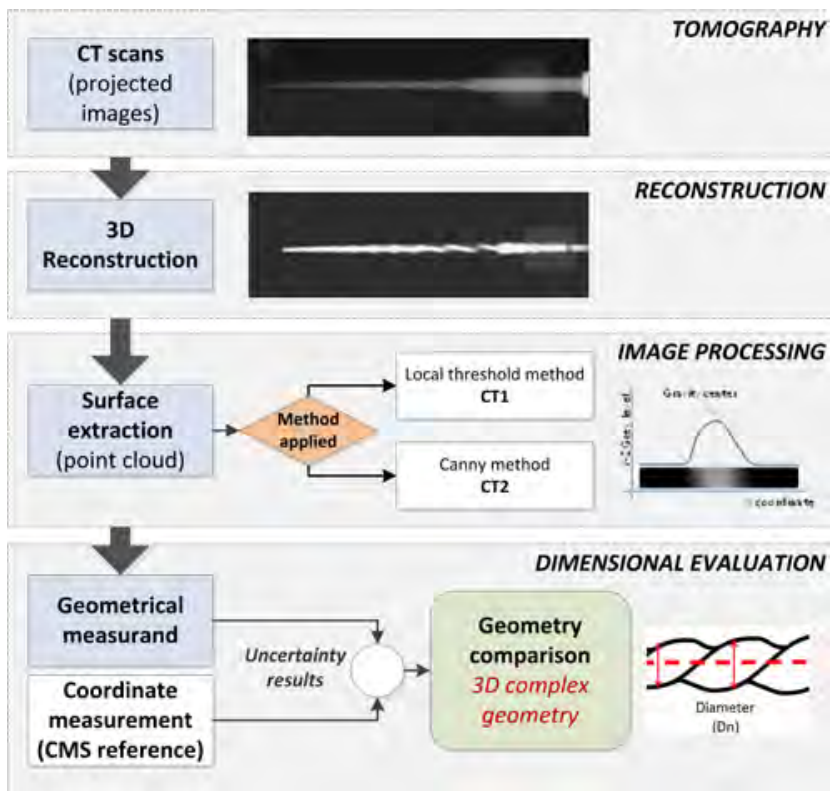

Figure 3. Workflow or process chain for CT measurement evaluation: case study of a miniaturized dental file with 3D complex geometry.

\subsection{Surface extraction techniques applied: Local threshold and 3D Canny algorithm}

Two different techniques were applied for the surface extraction to perform the measurements of the workpiece by computed tomography: CT1 or local threshold method [29] and CT2 based on the 3D Canny algorithm [31]. Both techniques have been already applied to common geometric primitives (basic geometric shapes and forms, e.g. lines, planes, spheres...). In this work, where they are applied to complex geometries, the point clouds obtained by each technique are processed using the same measurement protocol with Metrolog XG software by Metrologic Group (Meylan, France). The brief description of both techniques is included below.

\subsubsection{Local threshold method (CT1)}

The specific CT1 technique used in this case needs a correction by locally adapting the threshold value, as explained later. Threshold method for surface extraction in CT is a well-known technique adapted from the 2D image segmentation. It is based on the determination of a gray value (called threshold) used to distinguish one material to the other. Voxels with higher gray value than threshold are considered belonging to the part, and voxels with lower value are considered as air. After that, sub-voxel techniques based on a local 3D interpolation are used to determinate the surface points.

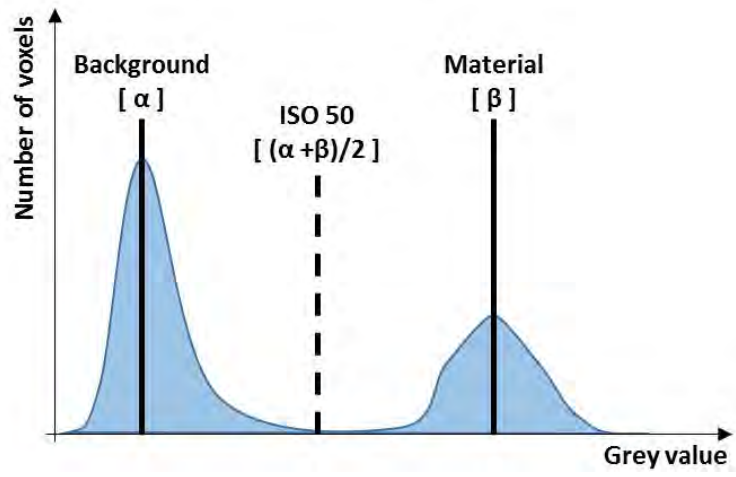

Figure 4. Determination of the threshold value based on the ISO50 method.

Threshold value can be determined using the ISO50 method [37]. This method is based on the determination of a reference gray value for each of the two materials, and the calculation of the 
ISO50 threshold value as their average. The reference value for each material is usually calculated as the peak value assigned to that material in the histogram graph (Figure 4). Although this method is widely used in multiple applications due to its simplicity, it does not guarantee an accurate determination of the surface $[22,28]$. Therefore, in this work, the threshold value obtained by the ISO50 method has been corrected. The correction method is based on finding, by an iterative process, a threshold value which minimizes the deviation between the reference value for $\mathrm{Dr}$ (obtained by an additional and more accurate tactile coordinate measuring system, a CMM with MРЕсмм $=2.3 \mu \mathrm{m}+(\mathrm{L} / 300) \mu \mathrm{m}, \mathrm{L}$ in $\mathrm{mm})$ and the measured value for $\operatorname{Dr}$ (see Caption Figure 2). A more detailed explanation of the whole process can be found in [29].

\subsubsection{Canny algorithm (CT2)}

Developed by the authors and implemented using the Matlab software by MathWorks (Natick, MA, USA), the named CT2 method is based on the 3D Canny algorithm [31] and its methodology is divided into four steps: (i) Preliminary surface detection, (ii) Sub-voxel resolution refinement, (iii) Measurement and (iv) Measurement correction.

\section{Preliminary surface detection}

A Gaussian filter is applied along each of the three Cartesian directions, using a $1 \times 10$ convolution mask oriented along the direction. After this phase, three different 3D images $(X-Y, Y-$ $\mathrm{Z}$ and $\mathrm{Z}-\mathrm{X}$ in Figure 5) are obtained, each showing the transition between materials along the corresponding direction.

\section{Sub-voxel resolution refinement}

A specific algorithm has been operated to calculate the points with XYZ-coordinates that define, with sub-voxel resolution, the material transition. This improves the actual spatial resolution of the edge detection method down to one hundredth of the voxel resolution. From the preliminary surface detected in the previous step, obtained from the calculated local maximum positions, a gravity center algorithm is applied to a neighborhood around each of those local maximum positions. The calculation of the optimal position of the point $\left(X^{\prime}, Y^{\prime}, Z^{\prime}\right)$ inside it with sub-voxel resolution is carried out by applying Eq.(1):

$$
X^{\prime}=\frac{\sum_{i=1}^{3}\left(X_{i} \cdot G_{X, i}\right)}{\sum_{i=1}^{3} G_{i}} ; \quad Y^{\prime}=\frac{\sum_{j=1}^{3}\left(Y_{j} \cdot G_{Y, j}\right)}{\sum_{j=1}^{3} G_{j}} ; \quad Z^{\prime}=\frac{\sum_{k=1}^{3}\left(Z_{k} \cdot G_{Z, k}\right)}{\sum_{k=1}^{3} G_{k}}
$$

being $X_{i}, Y_{j}$ and $Z_{k}$ the coordinates of the voxels inside the window, with $i, j$ and $k$ indicating the number of voxel, i.e. from 1 to 3 for the optimal neighborhood size calculated for this work (see Figure 5). $G_{x, i}, G_{Y, j}$ and $G_{z, k}$, with possible values from 0 to 65,535 (i.e. 16 bits), are the gray value transitions obtained in the preliminary surface detection phase for the $\mathrm{X}, \mathrm{Y}$ and $\mathrm{Z}$ directions, respectively. This refinement is carried out separately and independently along all the three XYZ directions obtaining the three different coordinates of each surface point. 

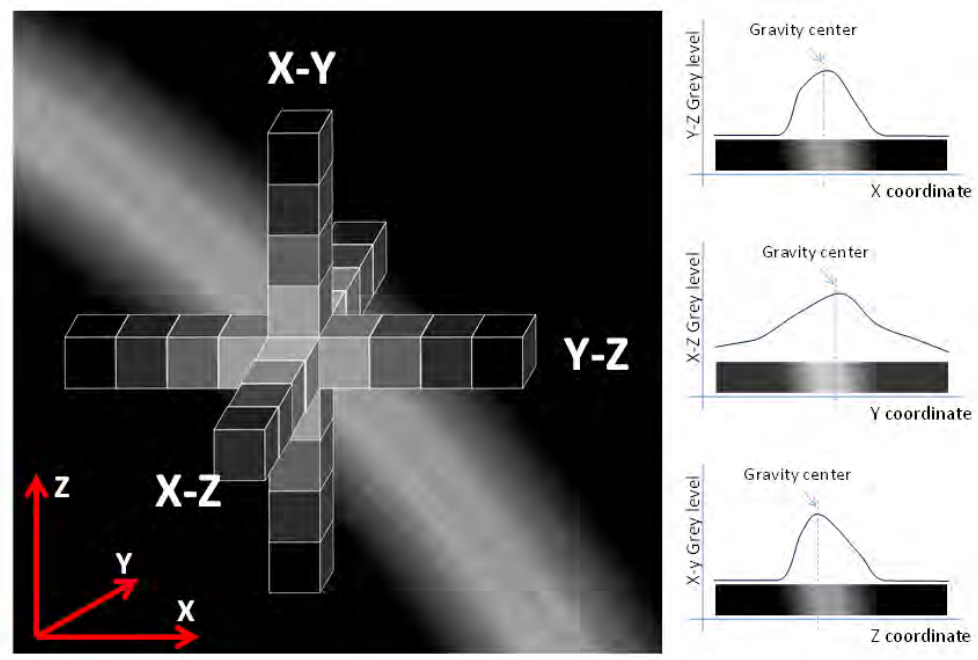

Figure 5. Sub-voxel resolution refinement (3D Canny algorithm, CT2).

\section{Measurement}

Using the point cloud of the part surface obtained from the previous step, coordinate measurements of the required dimensions can be carried out. This presents some challenges, especially for the 3D Canny algorithm since, as described above it uses an strategy that analyses the surface from the three main Cartesian directions in order to extract the point cloud. That was proven to be effective with regular surfaces than can be easily defined along those Cartesian axes [31]. However, in 3D complex geometries, which are not necessarily aligned with those Cartesian axes, the effectiveness of this algorithm is being analyzed in the present work.

\section{Measurement correction}

The correction applied in this work (Figure 6) includes the additional measurement of a specific parameter of the inspected part by another measuring technique (e.g. tactile or optical CMM). By comparing this result with the one obtained by the CT system a bias is calculated as a correction factor, which is applied to all the other measurements too. In the case presented the parameter used was again Dr (Figure 2) since it was simple to measure by a tactile CMM.

A more detailed explanation of the whole process can be found in [31].

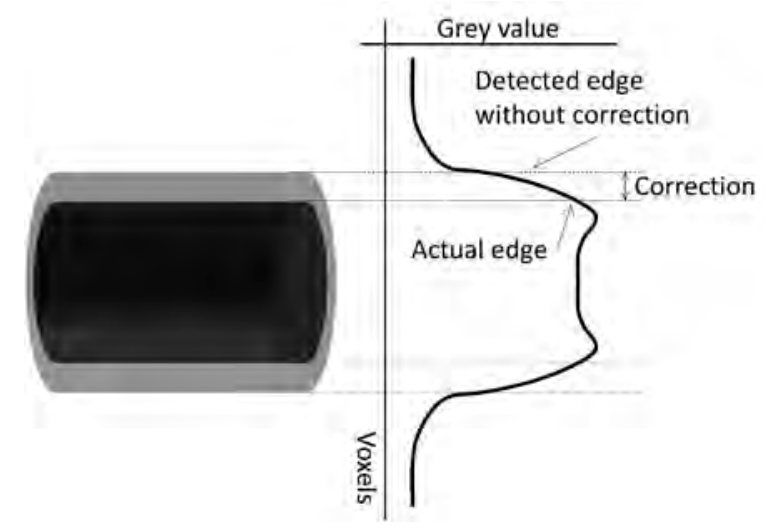

Figure 6. Measurement correction (3D Canny algorithm, CT2).

\subsection{Optical coordinate measurements}

Reference measurements of the endodontic file were performed on an optical coordinate measuring machine (OCMM) DeMeet 220 by Schut Geometrical Metrology (Groninge, The Netherlands) using a diascopic illumination with a light ring, a magnification lens $2 x$, an objective Numerical Aperture (NA) of 0.06 and a field of view of $3111 \mu \mathrm{m} \times 2327 \mu \mathrm{m}$. The uncertainty assessment of the OCMM measurements was carried out using a calibrated artefact. This artifact was 
a glass-chromium mask scale with an expanded calibration uncertainty of $\pm 0.5 \mu \mathrm{m}(\mathrm{k}=2)$. The OCMM uncertainty for length measurements in the 100-1000 $\mu \mathrm{m}$ range was evaluated, resulting in the maximum permissible error МРЕосмм $=1.7 \mu \mathrm{m}$ (i.e. suitable for the diameter measurements of the endodontic file). For the measurements of the endodontic file with a length $\mathrm{L}>1 \mathrm{~mm}$, the maximum permissible error of the OCMM obtained is: MPEосмм $=5 \mu \mathrm{m}+(\mathrm{L} / 150) \mu \mathrm{m}(\mathrm{L}$ in $\mathrm{mm})$.

The 3D complex geometry of the dental file has been measured by the OCMM and the CT. Since the OCMM is a $2 \mathrm{D}$ measuring system, the measurement repeatability has been evaluated considering different positions. A cube is firmly attached, using cyanoacrylate glue, to the workpiece at the bottom of the cutting area of the file, in order to use their faces as reference for the coordinate system (Figure 7a and Figure 10). Hence, the dental file measurement has been performed ten times for each of the four orientations, each one determined by the face of the cube resting parallel to the OCMM measuring stage (Figure $7 \mathrm{~b}$ ). Therefore, a direct comparison between the OCMM and the CT measurements for each of the four orientations can be carried out.

(a)

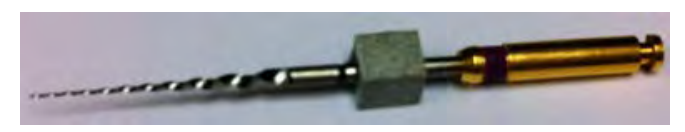

(b)

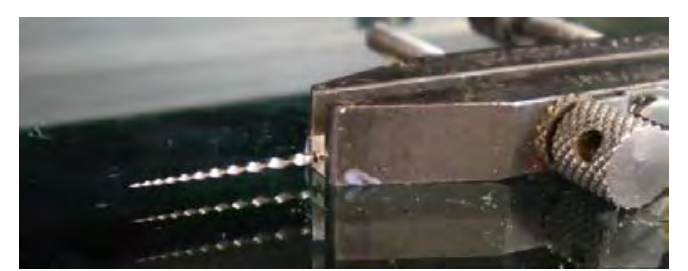

Figure 7. (a) Reference cube applied to the dental file; (b) dental file during the measurement on the OCMM.

\subsection{Computed Tomography scanning}

263

264

265

266

267

268

269

270

The dental file was scanned using a General Electric eXplore Locus SP by GE Healthcare (Chicago, IL, USA) cone-beam micro-CT machine. The reconstruction process was performed using the software provided by the manufacturer. The selected parameters used for the CT measurements were the presented in Table 1. During the scanning of the workpiece the temperature was continuously recorded inside the machine, obtaining a temperature range of $20 \pm 2^{\circ} \mathrm{C}$. As shown in Figure 8, a miniaturized ball-bar reference standard previously calibrated was also scanned with the dental file. This reference allowed the determination of the scale factor and the correction of the scale error of the measurements obtained.

Table 1. CT scanning parameters for the dental file workpiece.

\begin{tabular}{cc}
\hline Parameter & Value \\
\hline Voltage & $90 \mathrm{kV}$ \\
Current & $80 \mu \mathrm{A}$ \\
Increment angle & 0.4 degrees \\
Voxel size & $28 \mu \mathrm{m}$ \\
\hline
\end{tabular}

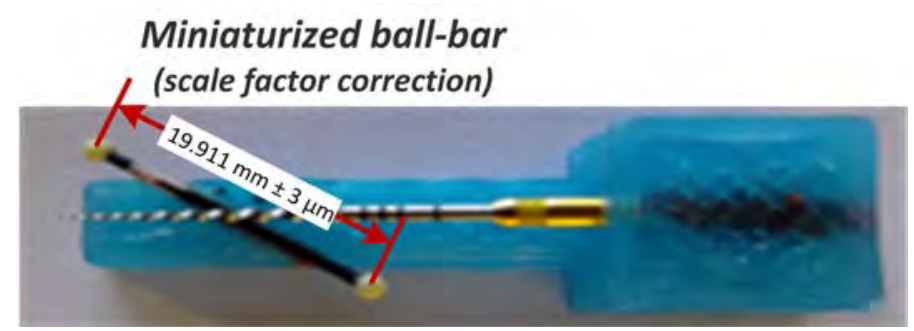

Figure 8. Dental file and miniaturized ball bar during the measurement on the CT scanner. 
An example of the points cloud obtained after the surface extraction process can be observed in Figure 9. Figure 9a shows the complete scan of the dental file (including the reference cube used for the alignment of the measurement). In Figure $9 \mathrm{~b}$ a detail of the dental file tip and of the 3D complex helix geometry is presented. Measurements are performed over the point cloud in order to avoid distortions caused by the surface reconstruction algorithms.

(a)

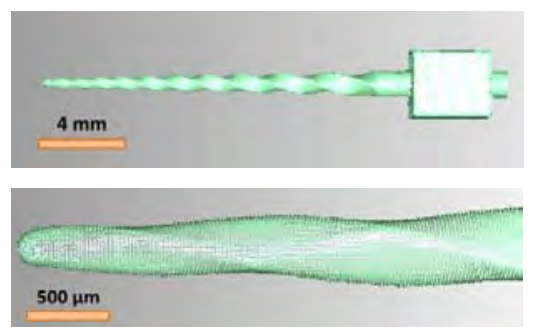

Figure 9. Point cloud from the CT scan of the file: (a) complete scan of the dental file; (b) detail of the dental file tip and of the helix geometry.

\subsection{Measurement strategy}

A common measurement procedure and reference coordinate system to be used by both measuring systems (i.e. OCMM and CT) was agreed. As it is introduced in subsection 2.4, it included the use of a cube attached to the file in order to use their faces as reference for the coordinates system (see Figure 7a). Since the OCMM is a 2D measuring system, the access to all surfaces to be measured was achieved by placing the dental file in those four different orientations. The dental file measurement by the CT scanner was reproduced using also the four cube faces orientations as reference planes. As a consequence, a direct comparison between the OCMM 2D measurements and the CT measurements results with the dental file in the same orientation could be performed in terms of measurement repeatability.

The reference coordinate system for the dental file is obtained by a plane (one of the cube faces), a straight line (the axial axis of the dental file) and a point as the origin of the XYZ-system. Firstly, the XZ-plane is created taking the superior face of the cube (see Figure 10a). Secondly, the main long $Z$-axis of the dental file is defined by joining the center of the spherical tip together with the center of the base cylinder (see Figure 10b). The spherical tip of the file measured was $0.06 \mathrm{~mm}$ in diameter. The cylinder is adjusted on the base of the endodontic file between the operative zone and the reference cube at a distance of $0.2 \mathrm{~mm}$ from both elements, respectively. Finally, the origin is defined as the intersection of the axial Z-axis and a plane measured on the cube face oriented to the dental file (see Figure 10c).

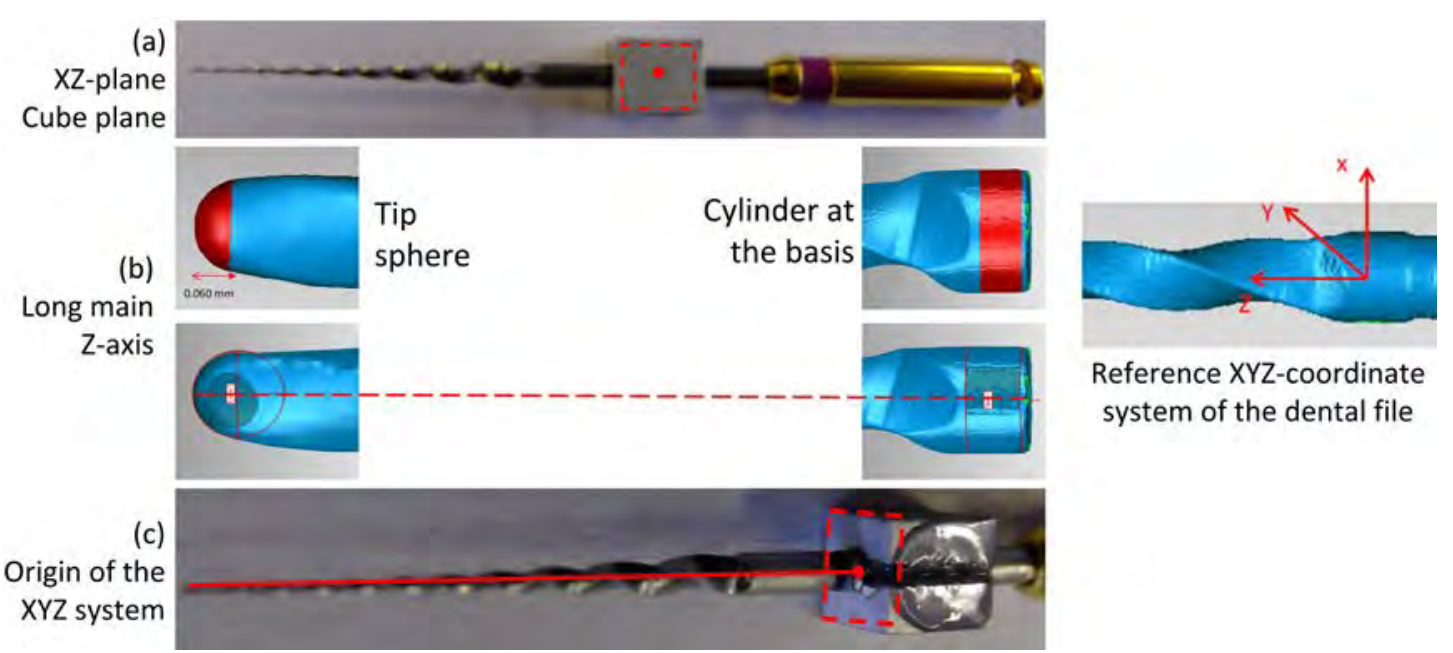

Figure 10. Measurement procedure of the dental file: (a) XZ-plane; (b) Z-axis; (c) XYZ-origin. 


\section{Measurement results and uncertainty estimation}

\subsection{Systematic error analysis}

In order to compare the two surface extraction methods the first influence analyzed is the systematic deviation of measurements by calculating the difference between the mean measurement performed using the CT scanner and the reference value obtained by the OCMM. To provide a comprehensive representation of all data for each type of measurand (diameters $\mathrm{D}_{0}$ to $\mathrm{D}_{12}$, helix pitches $\mathrm{P}_{1}$ to $\mathrm{P}_{9}$ and helix angles $\mathrm{H}_{1}$ to $\mathrm{H}_{9}$ ) (see Figure 11) the distribution of the systematic error results coming from the measurements taken at the four different orientations are used (one for every face of the base cube). Figure 11 illustrates the number of measured error results (frequency is indicated as bar heights, see $Y$ axis) in each error interval considered ( $X$ axis). It can be observed that the higher peaks (i.e. the higher number of measurement errors) tend to be centered in error intervals close to zero, which indicates that the surface extraction methods used minimize bias errors.

As it is shown in Figure 11a, Figure 11b and Figure 11c for diameter, helix pitch and helix angle measurement results, respectively, the systematic errors are substantially influenced by the employed surface extraction technique. In particular, the application of the CT2 technique (i.e. based on 3D Canny) allows obtaining a higher number of measurements closer to the calibrated values (higher bars close to zero) than when applying the CT1 technique (i.e. based on local threshold). On the one hand, by applying the CT1 method, reference values for most of the elements to be measured are usually needed in order to adjust the ISO factor [27], which could be particularly difficult when measuring 3D complex geometries, as in the presented work. On the other hand, the edge detection technique based on the Canny algorithm (CT2) provides a good edge location capability, less dependent on the geometry of the measured part. It might be corrected by using only one dimensional reference (Dr in this case). That significantly reduces the influence of the image quality, i.e. image noise. Results from both methods are similar in the helix angle case (Figure 11c). This is due to the fitting strategy for the determination of the tangent on the cutting edge and software applied, which results more influent than the bias error.

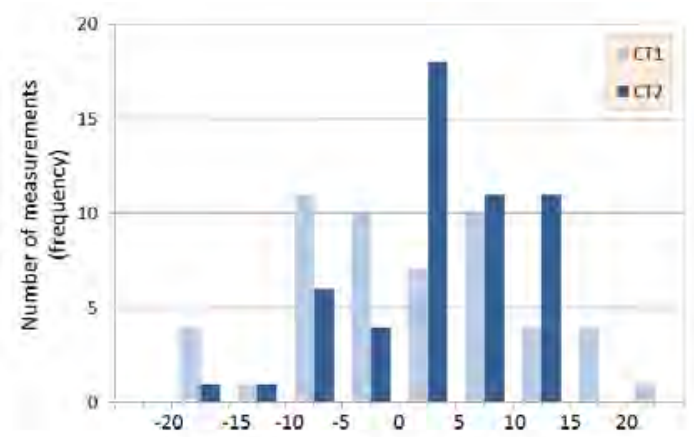

(a) Diameter (Dn) deviation from OCMM calibrated value $[\mu \mathrm{m}]$

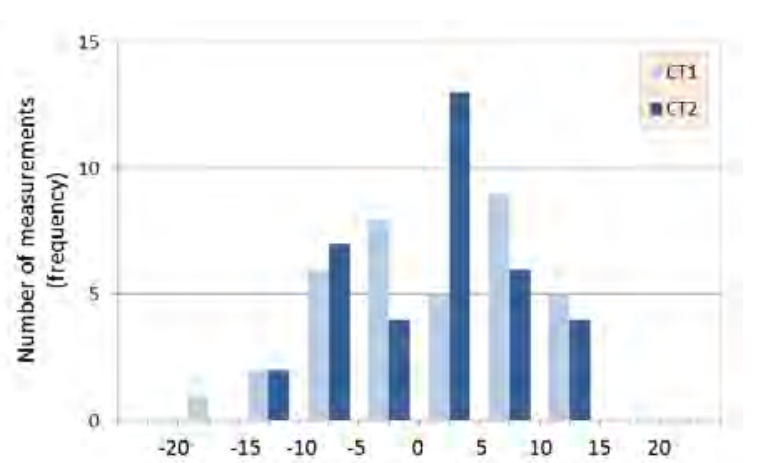

(b) Helix pitch (Pn) deviation from OCMM calibrated value [ $\mu \mathrm{m}]$

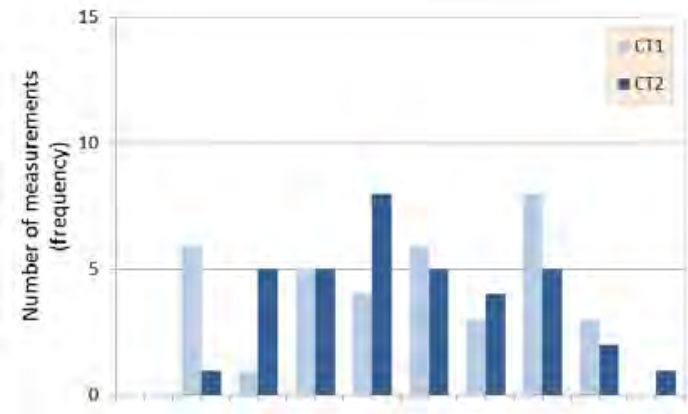

(c) Helix angle ( $\mathrm{Hn}$ ) deviation from OCMM calibrated value [deg] 
Figure 11. Deviations distribution of (a) diameter, (b) helix pitch and (c) helix angle measurands, obtained by CT system from OCMM calibrated values and applying CT1 and CT2 surface extraction techniques.

\subsection{Uncertainty estimation for optical CMM measurements}

Optical CMM measurements were used to validate the CT measurements. The measurement uncertainties for optical measurements with the OCMM were calculated according to ISO 14253-2 [38], considering two influence factors as described in equation (2):

$$
U_{95, \text { OCMM }}=k \sqrt{u_{c, \text { OCMM }}^{2}+u_{p, O C M M}^{2}}
$$

where $\mathrm{k}$ is the coverage factor ( $\mathrm{k}=2$ for a coverage interval of $95.45 \%)$, $\mathrm{u}_{\mathrm{c}, \mathrm{OCMM}}$ is the standard uncertainty of the OCMM based on the MPE of this measuring system ( $\left.u_{c, O C M M=M P E O C M M} / 2\right)$ and $\mathrm{u}_{\mathrm{p}, \mathrm{OCM}}$ is the standard uncertainty of the measuring procedure, i.e. standard deviation of the repeated measurements (repeatability, $n=10$ ). The OCMM is placed in a metrology laboratory with standard conditions of temperature, $20 \pm 1^{\circ} \mathrm{C}$ and humidity, $50-70 \%$.
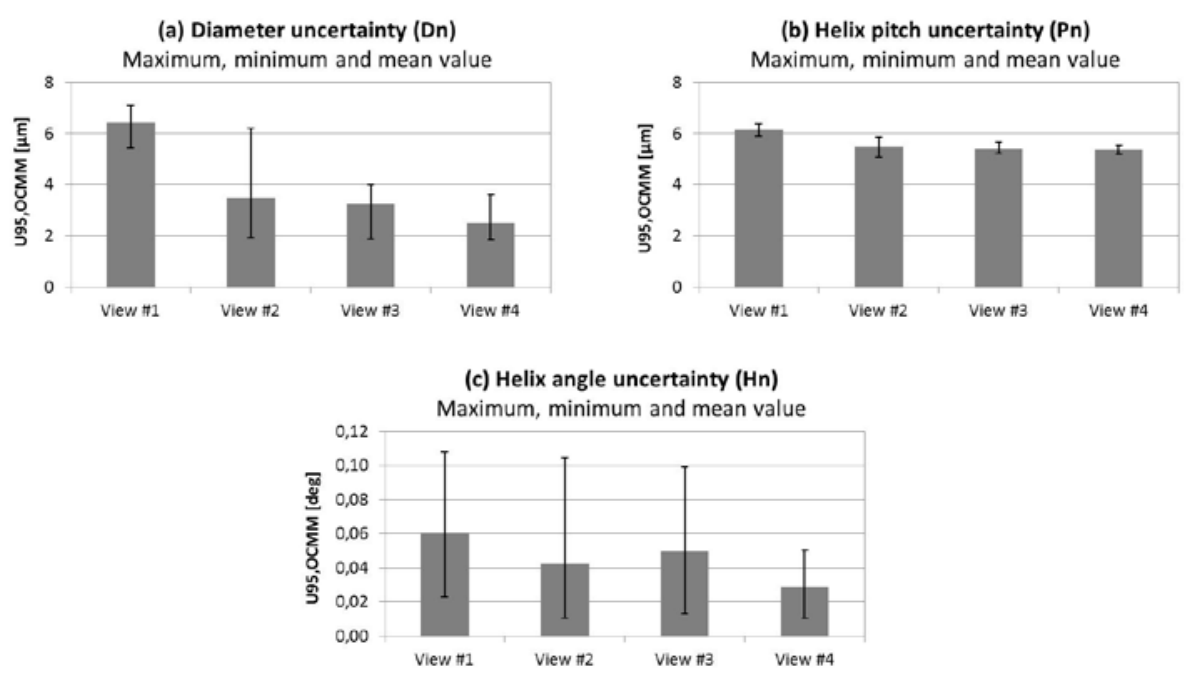

Figure 12. OCMM measurement uncertainty results of the four views and measurands (U95,OCMM): (a) Diameter, Dn; (b) Helix pitch, Pn; (c) Helix angle, Hn.

The results of the expanded uncertainty U95,Осмм were estimated for the four views and the four selected measurands: length (La), variable diameter (Dn), helix pitch (Pn) and helix angle (Hn). The angle measurement uncertainty was estimated by applying the error propagation law, as described in the GUM [39]. The maximum, minimum and mean uncertainty values obtained for all the parameters studied (Dn, Pn and Hn) are shown in Figure 12a, Figure 12b and Figure 12c, respectively. As it is illustrated, those values can be assumed as representative for each measurand of the whole workpiece. Thus, the maximum expanded uncertainty values from OCMM measurements are:

- $\mathrm{U}_{\mathrm{MAX}, \text { OCMm }}(\mathrm{La})=7.9 \mu \mathrm{m}$

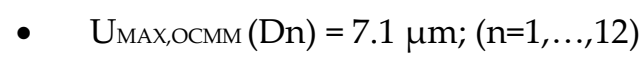

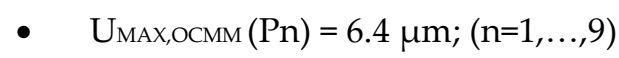

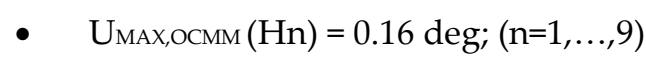

\subsection{Uncertainty estimation for CT measurements}

Measurement uncertainties for CT system were calculated. Despite of the lack of accepted test procedures and standards, numerous efforts have been focused on defining a fundamental 
document for specification and verification of CT systems used for coordinate metrology. As a result, several VDI/VDE guidelines are nowadays the main basis for the future development of ISO standards. The main tests to evaluate length measurement and probing errors are specified in VDI/VDE 2630-1.3 [40]; and influencing factors and a guide for the determination of uncertainty are described in VDI/VDE 2630-2.1 [41], the most applied procedure and recent guideline of task-specific calibration based on the substitution method. In some cases, when the substitution method is not applicable because a previous calibration with a more accurate system is unfeasible (as it is in this case), the uncertainty estimation can be achieved according to ISO 14253-2 [38], by considering the main error contributors in $\mathrm{CT}$, as shown in equation (3):

$$
U_{95, C T i}=k \sqrt{u_{r}^{2}+u_{p}^{2}+u_{w}^{2}+u_{b}^{2}}
$$

The term $\mathrm{k}$ is the coverage factor $(\mathrm{k}=2)$ and the $\mathrm{i}$-index $(\mathrm{i}=1,2)$ refers to the two surface extraction methods: CT1 (local threshold method) and CT2 (Canny algorithm) in order to obtain U95,CT1 and $\mathrm{U}_{95, \mathrm{CT} 2}$, respectively. The term $\mathrm{ur}_{\mathrm{r}}$ is the standard uncertainty due to traceability quantified by the MPE of the CT $\left(\mathrm{ur}_{\mathrm{r}}=\mathrm{MPE} \mathrm{CTi}_{\mathrm{i}} / 2\right)$, which are respectively: $\mathrm{MPE}_{\mathrm{cT} 1}=6.6 \mu \mathrm{m}+(\mathrm{L} / 5.4) \mu \mathrm{m}$; and MPEст2 $=7.0$ $\mu \mathrm{m}+(\mathrm{L} / 5.6) \mu \mathrm{m}$, where $\mathrm{L}$ is in $\mathrm{mm}$. These micro-CT system MPE expressions were experimentally determined by using several calibrated reference artefacts with maximum calibration uncertainties lower than $\pm 3.0 \mu \mathrm{m}$ for all the dimensions used. Additionally, $\mathrm{u}_{\mathrm{p}}$ is the standard uncertainty of the measurement procedure (repeatability), $\mathbf{u}_{\mathrm{w}}$ is the standard uncertainty from the material and manufacturing variations of the measured process, including the variations in the CTEs (coefficient of thermal expansion) of the workpiece, and $\mathrm{ub}$ is the standard uncertainty associated with the residual systematic error of the measurement process, which is influenced by the surface extraction technique (mainly dependent on the measurement correction, so that the standard uncertainty of the scale factor and the applied offset determination are here considered) and by the influence of the temperature variation during the $\mathrm{CT}$ measuring process.

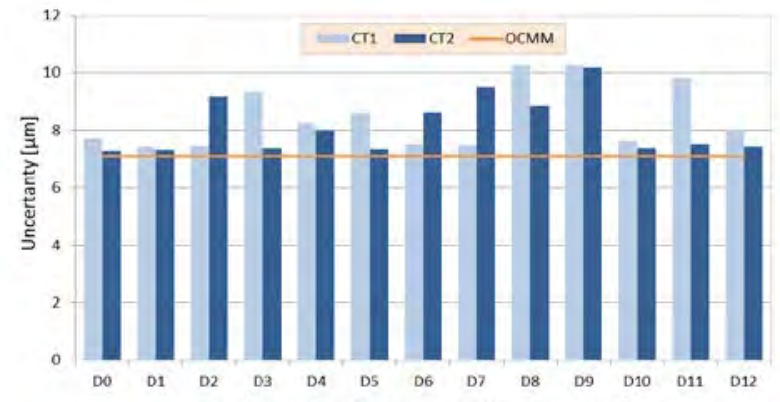

(a) Diameter (Dn) expanded uncertainty $U_{95, c t}$
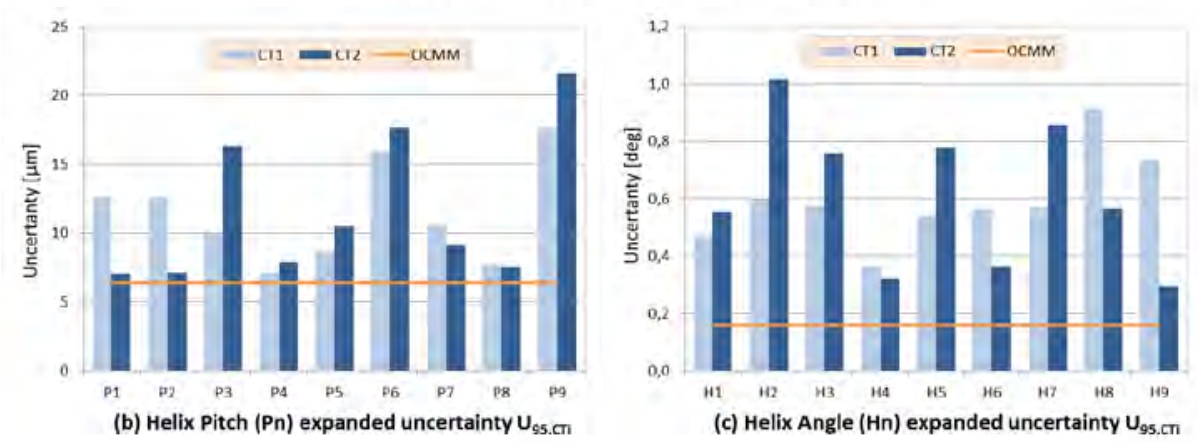

Figure 13. OCMM and CT expanded measurement uncertainty results (U95) of the four views and measurands: (a) Diameter, Dn; (b) Helix pitch, Pn; (c) Helix angle (Hn).

The comparison between the expanded uncertainty $\mathrm{U}_{95, \mathrm{CT} 1}, \mathrm{U}_{95, \mathrm{CT} 2}$ and $\mathrm{U}_{\mathrm{MAX}, \mathrm{OCMM}}$ is shown in Figure 13a, Figure 13b and Figure 13c for diameter, helix pitch and helix angle measurands, respectively. Considering the diameter, for smaller values of Dn the expanded uncertainty obtained by $\mathrm{CT}$ is closer than the considered Uмах,осмм. Since helix pitch and helix angle measurements 
strongly depend on the fitting procedure from the point cloud, these measurands present higher differences with respect to the reference OCMM value. If both surface extraction methods are compared, there are not clear differences between them in most of the measurands. On the other hand, in some cases CT1 shows lower uncertainties, while the opposite happens for some other measurands. In addition, for a further analysis of these results, the estimated uncertainties are eventually compared with the dental file's calibration and tolerances. Hence, in next subsection the EN value is calculated for all measurands and the $2 \mathrm{U} / \mathrm{T}$ ratio is also estimated to compare both extraction techniques when verifying 3D complex geometries in this micro manufactured part.

\subsection{EN value and tolerance verification capability}

To validate the expanded uncertainty results in relation to the measuring uncertainty of the used instruments, CT system and OCMM, the EN value was calculated for all measurands [42]. This parameter is given by equation (4) and relates the deviation between a measured value (i.e. by the $\mathrm{CT}$ systems in the present case) and the corresponding calibrated value (i.e. by the CMS) concerning their respective stated uncertainties. Then, if $\mathrm{EN}_{\mathrm{N}}<1$ there is a satisfactory agreement between the two values, otherwise there is no agreement among them.

$$
E_{N}=\frac{\left|\left(C T_{\text {meas.value }}\right)-\left(O C M M_{\text {ref.value }}\right)\right|}{\sqrt{U_{C T}^{2}+U_{\text {OCMM }}^{2}}}
$$

Figure 14 illustrates the percentage of $\mathrm{E}_{\mathrm{N}}<1$ values results for all the measurands of the dental file. As in the previous subsection, not significant differences between both techniques are observed. In general CT2 shows very similar or slightly better results, except for the measurand La or total length of the cutting segment. Nevertheless, the represented percentage of this parameter only considers that single parameter measured in the four orientations. For the rest of parameters more measurands are considered (nine in four orientations for Helix Pitch, nine in four orientations for Helix Angle, etc.). Again, this analysis cannot be considered conclusive in terms of defining which of both techniques provides a lower measurement uncertainty.

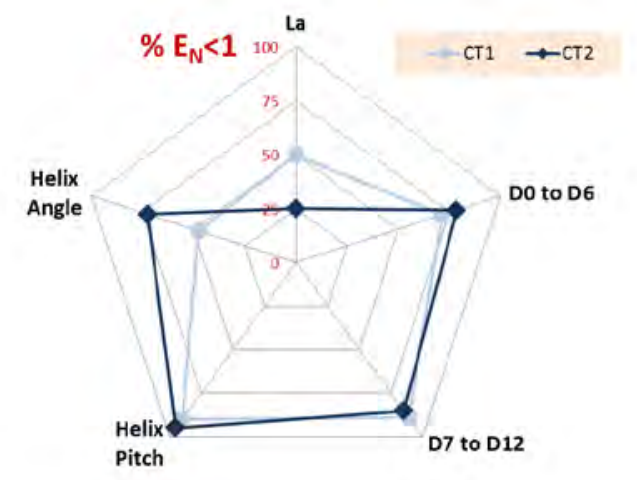

Figure 14. Percentage of $E_{N}<1$ values calculated for all CT measurement results and using both surface extraction techniques (CT1, CT2).

The ratio $2 \mathrm{U} / \mathrm{T}$ that considers the uncertainty measurement result $(2 \mathrm{U})$ and the tolerance of the workpiece (T) was analyzed. To assure the measuring capability of the CT system and the applied surface extraction techniques, the ratio must be $2 \mathrm{U} / \mathrm{T} \leq 0.4$, considering the micro-geometries of the dental file [41,43]. As previously presented for the EN value, the percentage of $2 \mathrm{U} / \mathrm{T} \leq 0.4$ values results are represented in Figure 15. Nevertheless, the measurands considered are only the length of the active cutting part (La) and variable diameters (D0 to D12), whose tolerance specifications were defined. As it is shown, the $100 \%$ of OCMM measurements meet the requirement. Both surface extraction methods CT1 and CT2 have also a high number of measurements that accomplish with the tolerance ratio specification: $78.6 \%$ and $85.7 \%$, respectively. The results provided for both methods are similar. Nevertheless, CT2 or Canny algorithm offers a slightly better performance 
according to the results shown in Figure 15. As a conclusion of the whole uncertainty assessment study and despite higher uncertainties and challenges in performing CT scanning metrology, the use of this technology for tolerance verification on complex geometries has been demonstrated to be adequate.

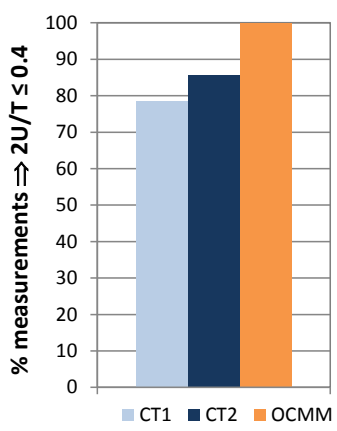

Figure 15. Percentage of $2 \mathrm{U} / \mathrm{T} \leq 0.4$ values calculated for all $\mathrm{CT}$ and OCMM measurement results with tolerance specification and using both surface extraction techniques (CT1, CT2).

\section{Conclusions}

In this paper a comparative analysis of two surface extraction techniques in computed tomography has been presented for the case study of a micro-component (a dental file) with 3D complex geometry. The contribution of the post-processing phase in CT dimensional measurements is here evaluated by applying the threshold determination strategy (CT1) and the Canny algorithm (CT2). Reference measurements were performed on an optical coordinate measuring machine (OCMM). Considering systematic errors results, it was found that the edge detection technique CT2 provides an edge definition with slightly lower systematic errors and, therefore, less dependent on the geometry of the measured part. Furthermore, the 3D Canny adapted method includes a direct correction instead of the iterative correction method of the local threshold, which simplifies its application. The uncertainty results do not show a clear difference between both techniques, although slightly better results have been observed for CT2 than for CT1, especially when the tolerance verification has been analyzed. Therefore, from this study both the threshold determination strategy and the 3D Canny technique show a similar behavior when tolerance is verified by performing CT scanning metrology on complex micro-geometries.

Consequently, regarding the 3D Canny algorithm it can be concluded that concerning accuracy and uncertainty it is, at least, as effective as the threshold technique when it is used for 3D complex micro-geometry dimensional measurements. This confirms the results obtained in [31] for regular and more simple geometries.

Particularly, since the 3D Canny adapted method includes a direct correction instead of the iterative correction method of the local threshold, its impact lies on the fact that once the point cloud generated by the CT system is calibrated with relatively low uncertainty, all the points of the cloud will be constrained in a position which is also determined with a relatively low systematic error (slightly lower with the Canny method than by using a thresholding technique) and uncertainty (similar with both techniques). Potentially, such calibration will be applicable and valid as well as to any relative position between different points of the cloud, leading to the result that virtually any measurement of complex and/or freeform geometrical features can be also performed with relatively low uncertainty. The metrological verification of this possibility is dependent on the availability of a calibrated freeform surface. On this regards, challenges are still present in the procedure for some complex measurands of the dental file such as the helix angle, and for geometrical characteristics with a critical measurand definition such as the length of the active cutting edge. Further research work will be focused on the establishment of a traceable and reproducible procedure for the calibration of miniaturized high accuracy freeform components in order to obtain a three-dimensional uncertainty assessment of CT measurements. 
Acknowledgments: The authors acknowledge the support of the Research Foundation MINECO (Spain) via project DPI2015-69403-C3-1-R and University of Zaragoza and Centro Universitario de la Defensa (Spain) via project UZCUD2016-TEC-09. The present research was carried out within a joint research program between the Department of Mechanical Engineering at DTU (Technical University of Denmark) and the Department of Design and Manufacturing Engineering at the University of Zaragoza (Spain). Collaboration from the Laboratory of Geometrical Metrology of DTU Mechanical Engineering is acknowledged in connection with the optical coordinate measurements.

\section{References}

1. Tosello, G.; Hansen, H. N.; Gasparin, S. Applications of dimensional micro metrology to the product and process quality control in manufacturing of precision polymer micro components. CIRP Ann. - Manuf. Technol. 2009, 58, 467-472.

2. Bos, E. J. C. Aspects of tactile probing on the micro scale. Precis. Eng. 2011, 35, 228-240.

3. Petz, M.; Tutsch, R.; Christoph, R.; Andraes, M.; Hopp, B. Tactile-optical probes for three-dimensional microparts. Measurement 2012, 45, 2288-2298.

4. Claverley, J. D.; Leach, R. K. A review of the existing performance verification infrastructure for micro-CMMs. Precis. Eng. 2015, 39, 1-15.

5. Mathia, T. G.; Pawlus, P.; Wieczorowski, M. Recent trends in surface metrology. Wear 2011, 271, 494-508.

6. Bešić, I.; Van Gestel, N.; Kruth, J.-P.; Bleys, P.; Hodolič, J. Accuracy improvement of laser line scanning for feature measurements on CMM. Opt. Lasers Eng. 2011, 49, 1274-1280.

7. De Chiffre, L.; Carmignato, S.; Kruth, J.-P.; Schmitt, R.; Weckenmann, A. Industrial applications of computed tomography. CIRP Ann. - Manuf. Technol. 2014, 63, 655-677.

8. Yu, J.; Lynn, R.; Tucker, T.; Saldana, C.; Kurfess, T. Model-free subtractive manufacturing from computed tomography data. Manuf. Lett. 2017, 13, 44-47.

9. Hermanek, P.; Carmignato, S. Porosity measurements by X-ray computed tomography: Accuracy evaluation using a calibrated object. Precis. Eng. 2017, 49, 377-387.

10. Villarraga-Gómez, H.; Lee, C.; Smith, S. T. Dimensional metrology with X-ray CT: A comparison with CMM measurements on internal features and compliant structures. Precis. Eng. 2018, 51, 291-307.

11. Heinzl, C.; Kastner, J.; Gröller, E. Surface extraction from multi-material components for metrology using dual energy CT. IEEE Trans. Vis. Comput. Graph. 2007, 13, 1520-1527.

12. Krämer, P.; Weckenmann, A. Multi-energy image stack fusion in computed tomography. Meas. Sci. Technol. 2010, 21, 45105.

13. Borges de Oliveira, F.; Stolfi, A.; Bartscher, M.; De Chiffre, L.; Neuschaefer-Rube, U. Experimental investigation of surface determination process on multi-material components for dimensional computed tomography. Case Stud. Nondestruct. Test. Eval. 2016, 6, 93-103.

14. Kiekens, K.; Welkenhuyzen, F.; Tan, Y.; Bleys, P.; Voet, A.; Kruth, J.-P.; Dewulf, W. A test object with parallel grooves for calibration and accuracy assessment of industrial computed tomography (CT) metrology. Meas. Sci. Technol. 2011, 22, 115502.

15. Xue, L.; Suzuki, H.; Ohtake, Y.; Fujimoto, H.; Abe, M.; Sato, O.; Takatsuji, T. A method for improving measurement accuracy of cylinders in dimensional CT metrology. Comput. Des. 2015, 69, 25-34.

16. Stolfi, A.; De Chiffre, L. 3D artefact for concurrent scale calibration in Computed Tomography. CIRP Ann. Manuf. Technol. 2016, 65, 499-502.

17. Andreu, V.; Georgi, B.; Lettenbauer, H.; Yague, J. A. Analysis of the error sources of a Computer Tomography Machine. In Proc. Lamdamap conference; 2009; pp. 462-471.

18. Kruth, J. P.; Bartscher, M.; Carmignato, S.; Schmitt, R.; De Chiffre, L.; Weckenmann, A. Computed 
tomography for dimensional metrology. CIRP Ann. - Manuf. Technol. 2011, 60, 821-842.

513 19. Hiller, J.; Reindl, L. M. A computer simulation platform for the estimation of measurement uncertainties in 514 dimensional X-ray computed tomography. Meas. J. Int. Meas. Confed. 2012, 45, 2166-2182.

515 20. Weckenmann, A.; Krämer, P. Assessment of measurement uncertainty caused in the preparation of measurements using computed tomography. In 19th IMEKO World Congress 2009; Quality Management and Manufacturing Metrology, University Erlangen-Nuremberg, Erlangen, Germany, 2009; Vol. 3, pp. 1787-1791. 21. Ferrucci, M.; Ametova, E.; Carmignato, S.; Dewulf, W. Evaluating the effects of detector angular misalignments on simulated computed tomography data. Precis. Eng. 2016, 45, 230-241.

22. Hiller, J.; Reindl, L. M. A computer simulation platform for the estimation of measurement uncertainties in dimensional X-ray computed tomography. Measurement 2012, 45, 2166-2182.

23. Ferrucci, M.; Leach, R. K.; Giusca, C.; Carmignato, S.; Dewulf, W. Towards geometrical calibration of x-ray computed tomography systems - a review. Meas. Sci. Technol. 2015, 26, 92003.

24. Müller, P.; Cantatore, A.; Andreasen, J. L.; Hiller, J.; De Chiffre, L. Computed tomography as a tool for tolerance verification of industrial parts. In Procedia CIRP; 2013; Vol. 10, pp. 125-132. 25. Müller, P.; Hiller, J.; Cantatore, A.; De Chiffre, L. A study on evaluation strategies in dimensional X-ray computed tomography by estimation of measurement uncertainties. Int. J. Metrol. Qual. Eng. 2012, 3, 107-115. 26. Stolfi, A.; Thompson, M. K.; Carli, L.; De Chiffre, L. Quantifying the Contribution of Post-Processing in Computed Tomography Measurement Uncertainty. Procedia CIRP 2016, 43, 297-302. 27. Hiller, J.; Hornberger, P. Measurement accuracy in X-ray computed tomography metrology: Toward a systematic analysis of interference effects in tomographic imaging. Precis. Eng. 2016, 45, 18-32. 28. Kraemer, A.; Lanza, G. Assessment of the Measurement Procedure for Dimensional Metrology with X-ray Computed Tomography. Procedia CIRP 2016, 43, 362-367. 29. Ontiveros, S.; Yagüe-Fabra, J. A.; Jiménez, R.; Tosello, G.; Gasparin, S.; Pierobon, A.; Carmignato, S.; Hansen, H. N. Dimensional measurement of micro-moulded parts by computed tomography. Meas. Sci. Technol. 2012, 23, 125401. improvement of dimensional measurements obtained from a conventional micro-CT cone beam machine. CIRP J. Manuf. Sci. Technol. 2013, 6, 143-148.

540 31. Yagüe-Fabra, J. A.; Ontiveros, S.; Jiménez, R.; Chitchian, S.; Tosello, G.; Carmignato, S. A 3D edge detection 541 technique for surface extraction in computed tomography for dimensional metrology applications. CIRP Ann. 542 Manuf. Technol. 2013, 62, 531-534.

543 32. Ontiveros, S.; Yagüe, J. A.; Jiménez, R.; Brosed, F. Computed Tomography 3D Edge Detection Comparative 544 for Metrology Applications. Procedia Eng. 2013, 63, 710-719.

545 33. Ruddle, C. J. The ProTaper endodontic system: geometries, features, and guidelines for use. Dent. Today $5462001,20,60-67$.

547 34. Ruddle, C. J. The ProTaper technique. Endod. Top. 2005, 10, 187.

548 35. ISO 3630-1:2008. Dentistry. Root-canal instruments. Part 1: General requirements and test methods 2008.

549 36. Stolfi, A.; Thompson, M. K.; Carli, L.; De Chiffre, L. Quantifying the Contribution of Post-Processing in 550 Computed Tomography Measurement Uncertainty. Procedia CIRP 2016, 43, 297-302.

551 37. Kiekens, K.; Welkenhuyzen, F.; Tan, Y.; Bleys, P.; Voet, A.; Kruth, J.-P. A test object for calibration and 552 accuracy assessment in X-ray CT metrology. Proc. IMEKO 10th Int. Symp. Meas. Qual. Control 2010, B6_86_1-4. 38. ISO 14253-2:2011. Geometrical product specifications (GPS). Inspection by measurement of workpieces and

554 measuring equipment. Part 2: Guidance for the estimation of uncertainty in GPS measurement, in calibration of 
555 measuring equipment and in product verifi 2011.

556 39. Guide to the Expression of Uncertainty in Measurement (GUM) 2008.

557 40. VDI/VDE 2630 Part 1.3 Guideline for the application of DIN EN ISO 10360 for coordinate measuring 558 machines with CT-sensors 2011.

559 41. VDI/VDE 2630 Part 2.1 Determination of the uncertainty of measurement and the test process suitability of 560 coordinate measurement systems with CT sensors 2015.

561 42. ISO/IEC 17043. Conformity assessment. General requirements for proficiency testing 2010.

562 43. ISO 286-2:2010. Geometrical product specifications (GPS). ISO code system for tolerances on linear sizes.

563 Part 2: Tables of standard tolerance classes and limit deviations for holes and shafts 2010. 